\title{
Two-Step CAPTCHA: Using a Simple Two Step Turing Test to Differentiate between Humans and Bots
}

\author{
Adarsh Baluni \\ University of Pune \\ S.K.N.C.O.E- Dept. of I.T. \\ Vadgaon (Bk.), Pune-41, India
}

\author{
Sayali Gole \\ University of Pune \\ S.K.N.C.O.E- Dept. of I.T. \\ Vadgaon (Bk.), Pune-41, India
}

\begin{abstract}
Growth of Internet and its usage has made web services prone to malicious threats by automated scripts or bots. "Completely Automated Public Turing Test to tell Computers and Humans Apart", commonly abbreviated as CAPTCHA, is a technique used by web services to differentiate between humans and bots.[1] Most of these techniques are based on recognizing the distorted images of alphanumeric texts that are often not easy to understand by the humans. We put forward a new idea of preventing automated attacks by bots, which asks users to pass through a simple two-step process of authentication. The first step involves recognizing an image from a set of images that best answers to the question associated with this step. The second step involves entering the values associated with the image selected so as to further nullify the probability of a bot attack.
\end{abstract}

\section{General Terms}

Authentication, Security, Algorithms

\section{Keywords}

CAPTCHA, two step authentication, Image based CAPTCHA, Web Security, Turing test.

\section{INTRODUCTION}

Most of the websites these days present users with images of distorted and tampered alphanumeric texts at the time of web registrations. Each of these images is actually a simple test to differentiate between humans and bots. The idea used here is that humans can easily identify the text in such an image whereas it is difficult to teach a bot to recognize characters written in graphics.[2] This test is called CAPTCHA.

CAPTCHA stands for "Completely Automated Public Turing Test to tell Computers and Humans Apart" which is based on Turing Test. The Turing Test, proposed by Alan Turing in 1950 , is way of determining whether machines can think like humans.[3] The Turing Test is used to exhibit a machine's ability to display intelligent behaviour equivalent to, or indistinguishable from, that of a human[4]. This test has three participants - two subjects and judge. One of the subjects is a person and the other is a computer. Both subjects are hidden from the view of the judge. They communicate with the judge via text-only channels. The role of the judge is to determine which text channel corresponds to the human and which corresponds to the computer. If the judge cannot determine this, then the computer passes the test.[6]
The term CAPTCHA (for Completely Automated Public Turing Test To Tell Computers and Humans Apart) was coined in 2000 by Luis von Ahn, Manuel Blum, Nicholas Hopper and John Langford of Carnegie Mellon University. At the time, they developed the first CAPTCHA to be used by Yahoo[5]

CAPTCHAs must satisfy three basic properties [9]. The tests must be

- $\quad$ Easy for humans to pass.

- $\quad$ Easy for a tester machine to generate and grade.

- Hard for a software robot to pass. The only automaton that should be able to pass a CAPTCHA is the one generating the CAPTCHA.

\section{NEED}

Many of the websites today hold sensitive data, some may require user to sign up at times, while some websites are designed to conduct polls etc. These websites are in constant threat of attacks by automated scripts or bots. It becomes really important to use a mechanism that can easily identify humans and prevent machine attack. CAPTCHA play a key role in recognizing this disparity.

\section{EXISTING METHEDOLOGIES}

There are mainly four categories of CAPTCHA [7]

1. Text based CAPTCHA: This type of CAPTCHA involves using a sequence of distorted characters with noise added to them. The user is required to enter the text having the same sequence of the distorted characters.

2. Audio CAPTCHA: The user is made to pass through a test which involves recognizing an audio task.

3. Image based CAPTCHA: This is a hard AI problem and very difficult to break. Image based CAPTCHA involve recognizing the image that answers the question asked.

4. Puzzle based CAPTCHA: The user is required to solve a simple puzzle of images or puzzle in images, which a bot is most likely to fail.[8]

\subsection{Text Based CAPTCHA}

Text-based CAPTCHA are most widely used and deployed CAPTCHAs since many years in major web sites. Text-based CAPTCHAs are easy to use and can provide strong security if properly designed. Text based CAPTCHAs are transformed or deformed images of dictionary words which are chosen at random. They can also be alpha-numeric words or words without dictionary meaning. The user's task is to type the letters in the correct sequence as given in the transformed image in the space (a text box) provided. 


\section{$T V 37 C$}

Figure 3. 1 A simple Text Based CAPTCHA

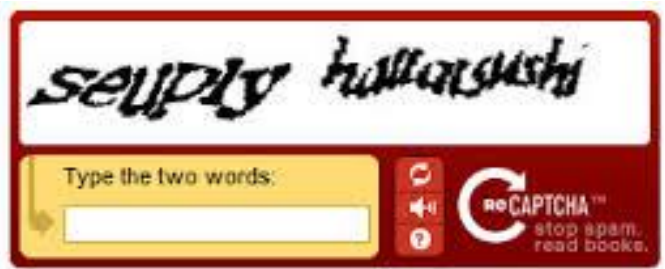

Figure 3. 2 Text-based CAPTCHA

Early text-based CAPTCHAs were straight forward for humans to solve. Advances in OCR techniques and consequently efficiency of bots in breaking text-based CAPTCHAs improved as a result of which text-based CAPTCHAs are designed harder. Hence, humans are finding it difficult to encode the CAPTCHA which in result is reducing the efficiency and also at the same time increasing the time required to solve the CAPTCHA.[10]

\subsection{Audio CAPTCHA}

Audio CAPTCHAs randomly take a sequence drawn from recordings which has simple words or numbers, combine them and add some disturbance to it so the audio is played in garbled noise. The CAPTCHA system then asks the user to enter the words or numbers in the recording accordingly. Audio CAPTCHAs are more difficult to solve, more demanding in terms of time and efforts in comparison to text and image CAPTCHAs. However, audio based CAPTCHA tests have become an alternative for visually impaired people.

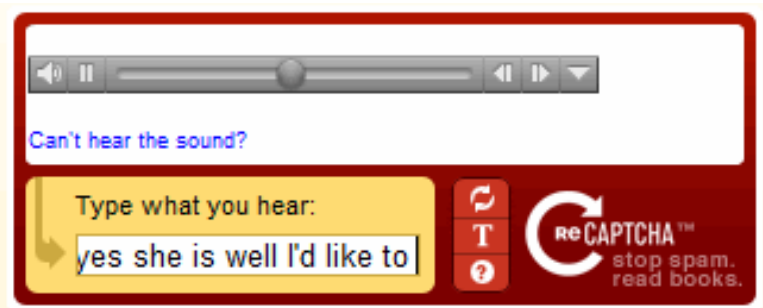

Figure 3. 3 Audio CAPTCHA

\subsection{Image based CAPTCHA}

Image-based CAPTCHA schemes have been proposed as an alternative to text-based CAPTCHAs and also audio based CAPTCHAs but they have not been successful in replacing text-based CAPTCHAs.

In this, a user is required to understand the problem statement and identify image/images that answer the problem statement. [10]

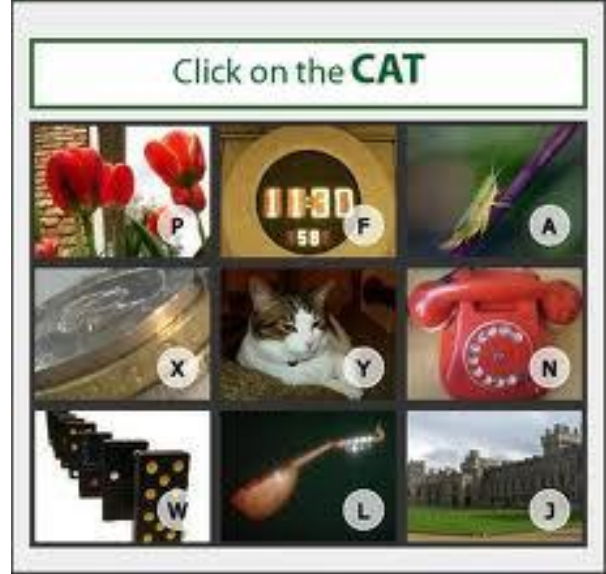

Figure 3. 4 An Image based CAPTCHA

The advantage of image based CAPTCHA is that pattern recognition is a hard AI Problem .this makes it is difficult to break this test using pattern recognition technique.[3]

\subsection{Puzzle based CAPTCHA}

In this test, a small mathematical problem is generated according to some predefined rules. The answer to the question is then cross checked with the database answer. Solving of this problem requires an ability of understanding text of question, only a human user can answer this question.[10]

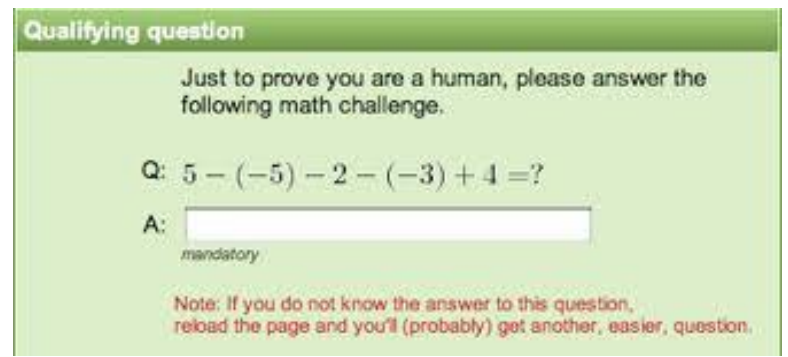

Figure 3. 5 Puzzle based CAPTCHA

Also, the shortcoming lies in the limit of the number of such questions that have a unique answer and that too which are easily recallable.

\section{OUR APPROACH}

The proposed methodology uses the idea of asking the user to go through a two-step process of authentication ( fig 4.1). In step one, a $3 \times 3$ grid of images is shown along with a question. The grid is surrounded with randomly chosen alphanumeric characters as shown in the fig 4.1. This question can be answered by only one image correctly out of the 9 images in the matrix. It requires user to simply click on the image which the user thinks is the answer. Once the user clicks on an image, the alphanumeric values associated with the image chosen appear as highlighted. The user then has to select the four values (top, bottom, left, right) in the corresponding drop down menus as shown in fig 4.1. After the values are entered, the user clicks on the 'submit' button. The user is authenticated only if the image chosen is correct as well the all the four values entered are correct.

It implies from this method that the probability of a bot passing this Turing test is extremely low. The probability of selecting a correct image is $1 / 9$. After selecting the image the user has to further enter one value in each of the four drop down menus. Each of the drop-down menus has 36 possible 
values (26 alphabets and 10 numeric values $0-9$ ). Only one is correct. So the probability of selecting a value in drop down menu is $1 / 36$. If we calculate the total probability of passing the Turing Test correctly, it comes out to be, $1 /(9 * 36 * 36 * 36 * 36)$ which is $1 / 15116544$. This is an extremely low probability for a bot to pass the test.

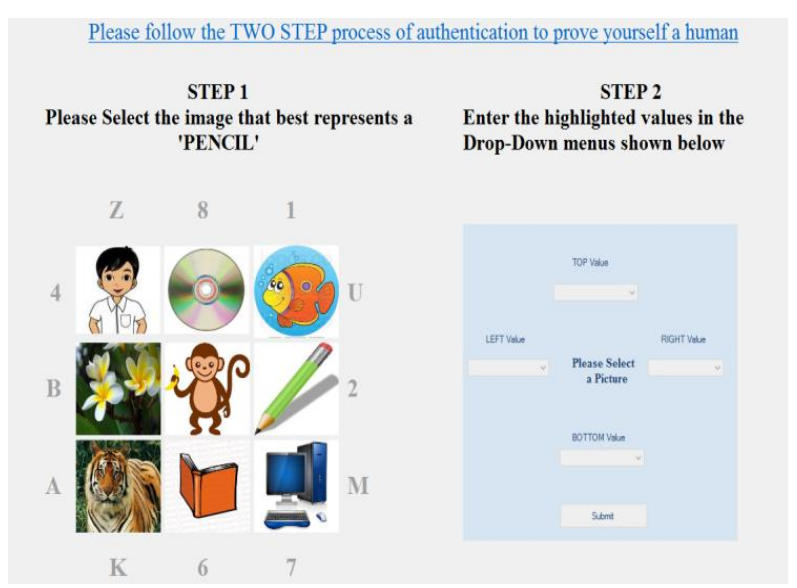

Figure 4.1 Two-step CAPTCHA

\subsection{Step by step Snapshots of the proposed methodology}

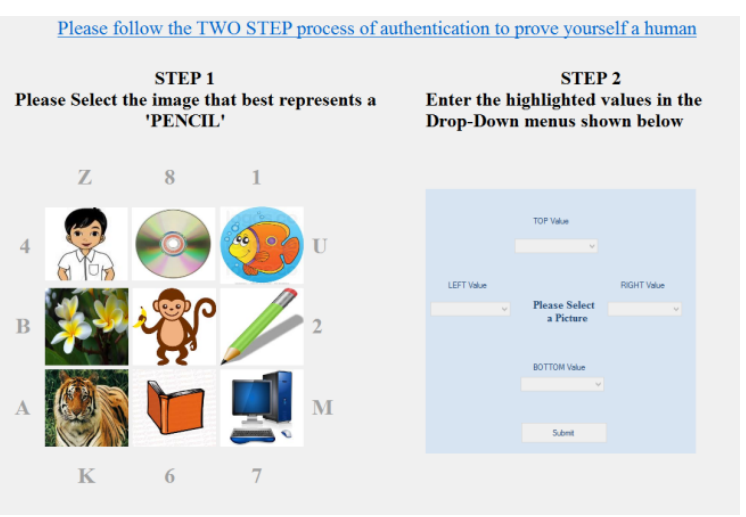

Figure 4.1.1 The screen at the start of the test

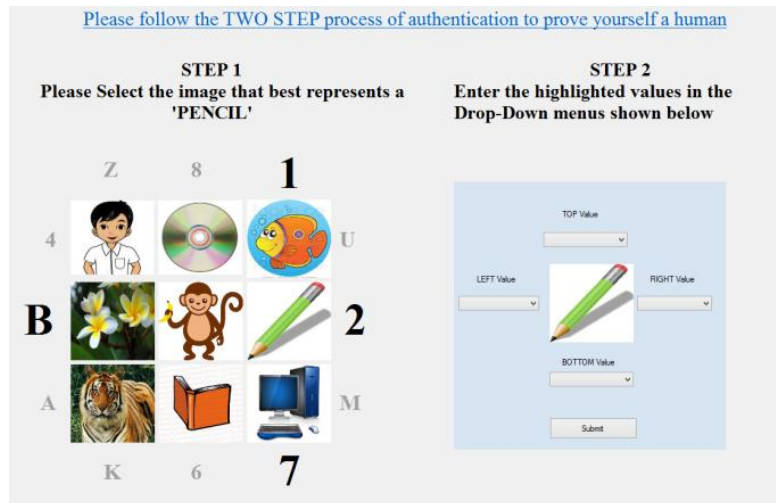

Figure 4.1.2 the image is clicked on and the highlighted values

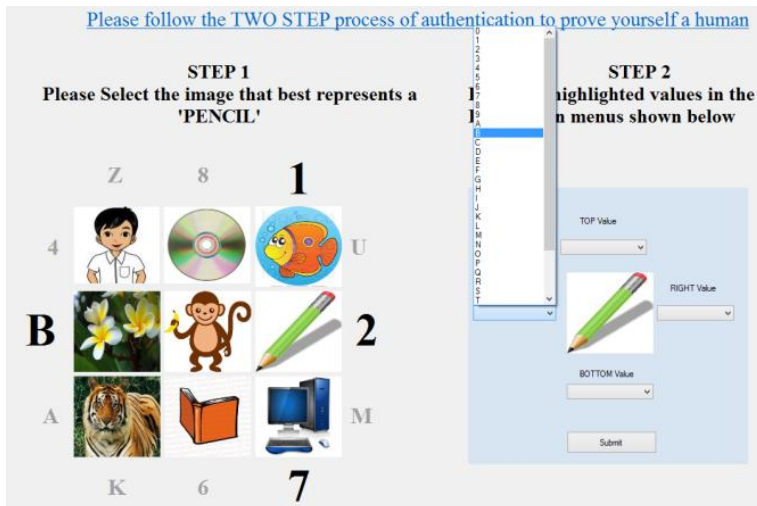

Figure 4.1.3 User entering the values in the drop-down menus

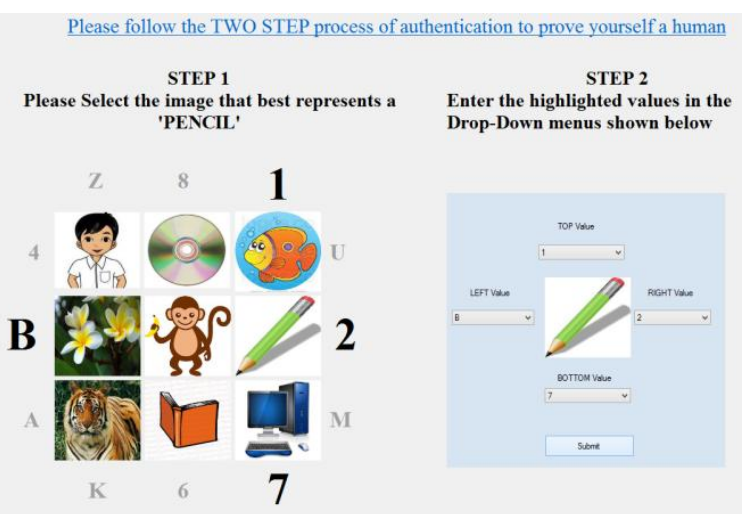

Figure 4.1.4 The image selected and the four values entered

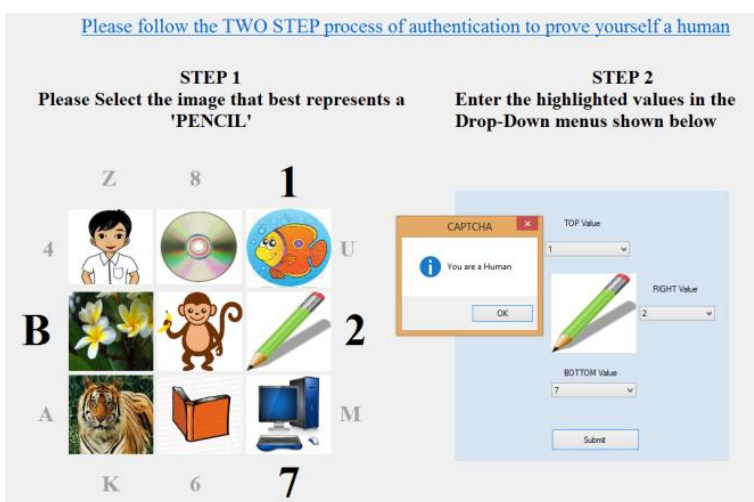

Figure 4.1.5 the user clicks on 'Submit' and the resultant message box. 


\subsection{Flowchart}

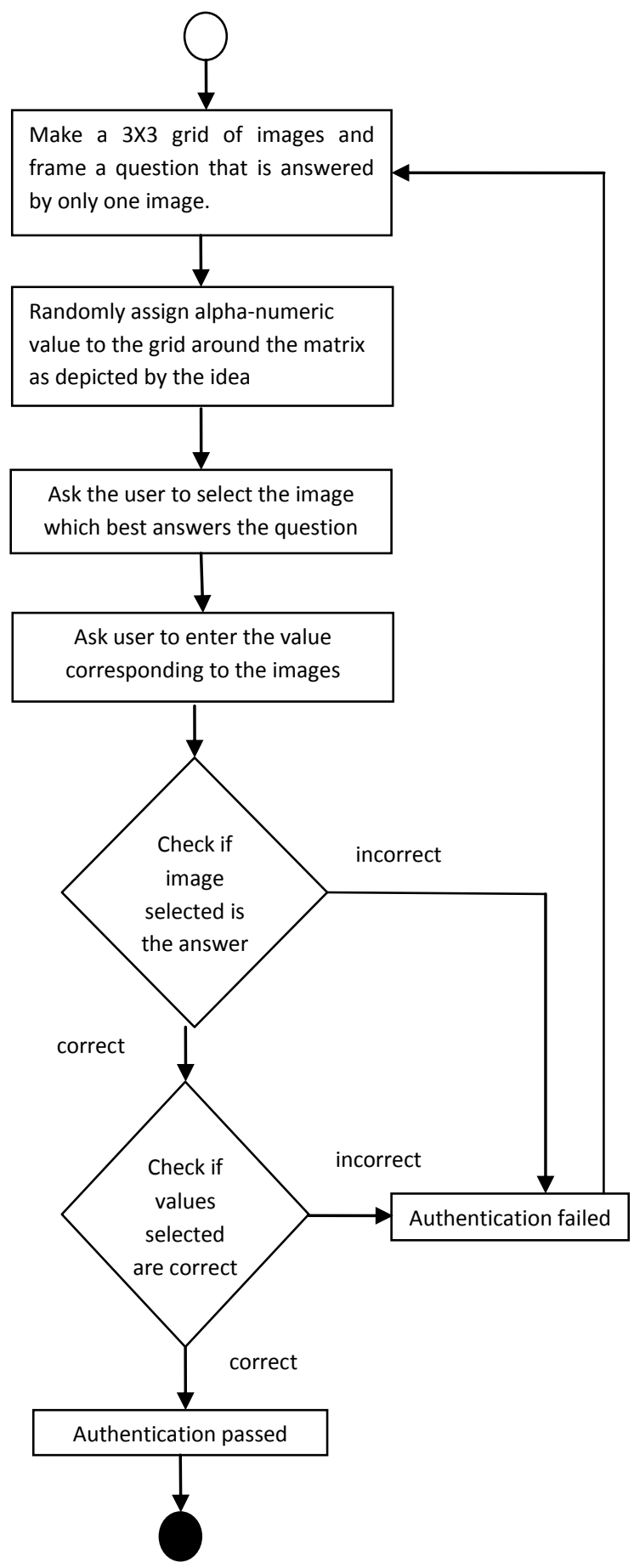

\subsection{Survey and Results}

To get a better understanding of its efficiency, we built an application on the proposed ides and surveyed around 300 people from technical and non technical background. We carried out systematic study on this proposed Two Step CAPTCHA methodology and came up with following results.

- $\quad 98 \%$ people surveyed preferred the proposed system over the conventional text-based CAPTCHA.

- As user got familiar with the proposed methodology, the response to pass the Turing Test time dropped from 30 seconds in the $1^{\text {st }}$ attempt to 15 seconds in the $5^{\text {th }}$ attempt

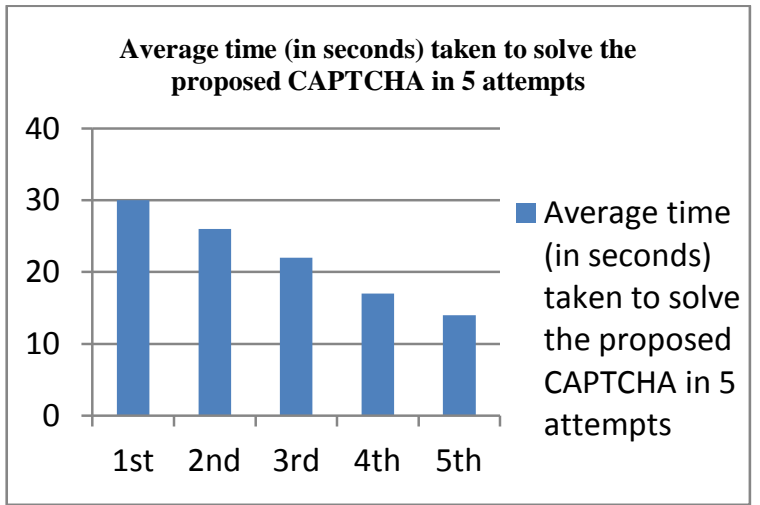

\section{CONCLUSION}

In the proposed method, the probability of the machine breaking unauthentically into websites is considerably low. Also at the same time the approach is simple and at times less time consuming. This approach overcomes the drawbacks of text-based and audio-based CAPTCHAs significantly and adds to the benefits of an image based CAPTCHA. The proposed system aims at effective handling of the security issues a website is vulnerable to.

\section{REFERENCES}

[1] R. Rehmam, D. Tomar and S. Das, "Dynamic Image Based CAPTCHA" , (2012) 978-0-7695-4692-6/12, IEEE Computer Society.

[2] Luis von Ahn, Manuel Blum and John Langford. Telling Humans and Computers Apart Automatically. In Communications of the ACM.

[3] Stanford Encyclopedia of Philosophy, http://plato.stanford.edu/entries/turing-test/

[4] Wikipedia: the free Encyclopedia, http://en.wikipedia.org/wiki/Turing_test.

[5] reCAPTCHA- http://www.google.com/recaptcha/captcha

[6] Montree Imsamai and Suphakant Phimoltares, "3D CAPTCHA: A next Generation of CAPTCHA.”, 2010 IEEE

[7] Chandvale, A.A; Sapkal, A.M; Jalnekar, R. M., A Framework to analyze the security of Text based CAPTCHA, International Journalof Computer Applications, Vol 1 issue 27, pp. 127-132.

[8] Haichang Gao, Dan Yao, Honggang Liu, Xiyang Liu, Liming Wang, "A Novel Image Based CAPTCHA Using Jigsaw Puzzle" 2010 IEEE Computer Society.

[9] Monica Chew and J. D. Tygar, UC Berkeley, "Image Recognition CAPTCHAs" Springer, September 2004, pp. 268-279.

[10] M. Tariq Banday, N. A. Shah, "A Study of CAPTCHAs for Securing Web Services" International Journal of Secure Digital Information Age, Vol. 1. No. 2, December 2009.

[11] Rizwan Ur Rahman "SURVEY ON CAPTCHA SYSTEMS",Journal of Global Research in Computer Science. 